\title{
A CHARACTERIZATION OF SPHERICAL SERIES REPRESENTATIONS OF THE FREE GROUP
}

\author{
GABRIELLA KUHN AND TIM STEGER
}

(Communicated by Jonathan M. Rosenberg)

\begin{abstract}
The spherical series representations of a free nonabelian group are here characterized as those irreducible unitary representations possessing a certain type of matrix coefficient. We make some conjectures on the problem of finding a natural boundary realization for a general irreducible representation of the free group, supposed to be weakly contained in the regular representation.
\end{abstract}

\section{StATEMENT OF THE Result AND Discussion}

The theory of unitary representations of nonabelian free groups is difficult and little developed. To represent the free group with two generators on a Hilbert space, you need only choose two unitary operators on the space. Moreover, common sense suggests that this representation will be irreducible for virtually all choices of the two operators. A free group is not type I, which means that the usual program of representation theory, decomposing unitary representations into irreducibles, is almost meaningless, because these decompositions will not usually be unique, or even close to unique. In fact, the little work which has been done is along the lines of producing and studying particular irreducible representations [Yoshizawa, Pytlik, Figà-Talamanca-Picardello 1 and 2, Kajiwara, Pytlik-Swarc, Figà-Talamanca-Steger 1 and 2, Cowling-Steger, Angelini, Steger].

Of these many examples, perhaps the clearest and most attractive is the spherical series of Figà-Talamanca and Picardello. Fix once and for all the free group $\Gamma$ and a set of free generators $\left\{a_{j}\right\}_{j=1}^{r}$. It is convenient to set

$$
q=2 r-1 \text {. }
$$

Construct a tree $\mathscr{T}$ with vertex set equal to $\Gamma$ saying that $x, y \in \Gamma$ have an edge between them if and only if $x=y a_{j}$ or $y=x a_{j}$ for some $j$. Then $\Gamma$ acts on the left on this tree as a subgroup of $\operatorname{Aut}(\mathscr{T})$, the full automorphism group of $\mathscr{T}$. If we give $\operatorname{Aut}(\mathscr{T})$ its natural topology as a subspace of the power space

Received by the editors April 4, 1990.

1980 Mathematics Subject Classification (1985 Revision). Primary 22D10, 22E45, 43A65; Secondary 20E05, 22E40.

Key words and phrases. Free group, unitary representation, spherical series. 
$\operatorname{Map}(\mathscr{T}, \mathscr{T})$, then $\operatorname{Aut}(\mathscr{T})$ is type $\mathrm{I}$ and its unitary representation theory is understood (see [Ol'shanskii 1 and 2]).

The subgroup $K_{0}$ of $\operatorname{Aut}(\mathscr{T})$ consisting of all automorphisms of $\mathscr{T}$ which fix the vertex $e$ is a maximal compact subgroup. The spherical series of $\operatorname{Aut}(\mathscr{T})$ consists of all irreducible unitary representations of $\operatorname{Aut}(\mathscr{T})$ which contain $K_{0}$ fixed vectors. (The spherical series is normally divided into a principal and a complementary series.) The spherical series of $\Gamma$ consists of the restrictions to $\Gamma$ of the spherical series of $\operatorname{Aut}(\mathscr{T})$.

The spherical series representations of $\Gamma$ are irreducible, as proved (for almost everyone of them) in [Pytlik], and slightly later (for every one of them) in [Figà-Talamanca-Picardello 1]. Moreover they are all inequivalent ([FigàTalamanca-Steger 2] contains a correct proof of this result of Figà-Talamanca and Picardello).

If we pass to a new set of generators $\left\{a_{j}^{\prime}\right\}$ by interchanging the $\left\{a_{j}\right\}$, or by replacing some of them with their inverses, or by applying conjugation, or by some combination of these operations, we arrive at an equivalent spherical series. However, any other change of generators for $\Gamma$ gives a spherical series of representations inequivalent to those of the original spherical series. (One can prove this using the techniques of [Bishop-Steger] and Theorem 3.7 of [CullerMorgan].)

This paper gives a characterization of the spherical series of $\Gamma$ :

Theorem. Let $\pi$ be an irreducible unitary representation of $\Gamma$ on the Hilbert space $\mathscr{H}$. Let $v_{0}, v_{1} \in \mathscr{H}$, let

$$
\phi=\left\langle v_{0}, \pi(x) v_{1}\right\rangle
$$

be a nonzero matrix coefficent of $\pi$, and consider $\phi$ as a function of the vertices of $\mathscr{T}$. Suppose that $\phi$ is invariant with respect to the subgroup

$$
K_{N}=\{g \in \operatorname{Aut}(\mathscr{T}) ; g x=x \text { whenever } d(x, e) \leq N\}
$$

for some $N \geq 0$. Then $\pi$ belongs to the spherical series.

Keep in mind that the choice of generators, $\left\{a_{j}\right\}_{j=1}^{r}$, enters in the definition of the tree $\mathscr{T}$ and that the spherical series also depends on the choice of generators. There are many unitary representations of $\Gamma$ which are proved to be irreducible (see the references in the first paragraph), and the total collection of irreducible unitary representations is, in imprecise terms, unmanageably huge. In spite of its special hypotheses, the above theorem is effective in that it picks the spherical series out of this huge collection.

Why bother to state or to prove this theorem? Because it is an exemplar of the general philosophy that irreducible unitary representations of $\Gamma$ are naturally realized as acting on spaces of functions defined on the boundary of $\Gamma$. Let

$$
\Omega^{\prime}=\left\{\left(x_{j}\right)_{j=0}^{\infty} ; x_{j} \text { is a vertex of } \Gamma \text { and } d\left(x_{j}, x_{j+1}\right)=1\right\},
$$


the set of half infinite geodesics in $\Gamma$. Say that $\left(x_{j}\right)$ and $\left(y_{j}\right)$ are equivalent if, up to some shift of the $\left(x_{j}\right)$, the two sequences of vertices are equal for all large enough $j$.

Then $\Omega$, the boundary of $\mathscr{T}$, is defined as the set of equivalence classes. Each equivalence class has a unique representative which starts at the point $e$, and this representative is of the form $\left(e, a_{i_{1}}^{\varepsilon_{1}}, a_{i_{1}}^{\varepsilon_{1}} a_{i_{2}}^{\varepsilon_{2}}, \ldots, a_{i_{1}}^{\varepsilon_{1}} \cdots a_{i_{j}}^{\varepsilon_{j}}, \ldots\right)$ where $1 \leq i_{j} \leq r, \varepsilon_{j}= \pm 1$, and if $i_{j}=i_{j+1}$, then $\varepsilon_{j}=\varepsilon_{j+1}$. It is natural to denote this point of the boundary by the infinite reduced word $a_{i_{1}}^{\varepsilon_{1}} a_{i_{2}}^{\varepsilon_{2}} a_{i_{3}}^{\varepsilon_{3}} \ldots$. We also call $\Omega$ the boundary of the free group $\Gamma$. Note that $\Omega$ does not depend essentially on the choice of generators; the boundary associated to any other choice of generators is naturally isomorphic to $\Omega$. The topology on $\Omega$ is that of the space of half-geodesics starting at $e$ considered as a subspace of the power space $\operatorname{Map}(\{j \in \mathbf{Z} ; j \geq 0\}$, vertices of $\mathscr{T})$. This makes $\Omega$ compact, in fact isomorphic to the Cantor set.

So far as the authors are aware, every single irreducible unitary representation of $\Gamma$ thus far constructed, which has the additional property of being weakly contained in the regular representation, is realized, and realized in a seemingly natural way, as acting on some space of functions defined on $\Omega$. For example, if $\nu$ is the unique regular measure on $\Omega$ invariant with respect to $K_{0}$, the representations $\pi_{s}$ of the spherical principal series of $\operatorname{Aut}(\mathscr{T})$ are given by

$$
\left[\pi_{s}(g) F\right](\omega)=F\left(g^{-1} \omega\right)\left|\frac{d \nu\left(g^{-1} \omega\right)}{d \nu(\omega)}\right|^{\frac{1}{2}+i s} .
$$

Here $s$ is real and may be taken in the range $[0, \pi / \log q]$. The $K_{0}$-invariant vectors of $\pi_{s}$ are the constant functions. The restrictions of the $\pi_{s}$ to $\Gamma \subseteq$ $\operatorname{Aut}(\mathscr{T})$ give us the representations of the spherical principal series of $\Gamma$. The complementary series representations are defined by the same formula, with $\operatorname{Re}(s)=0$ or $\operatorname{Re}(s)=\pi / \log q$ and $\operatorname{Im}(s)$ in the range [0,1/2]. The complementary series representations do not act on $L^{2}(\Omega, \nu)$ but on certain Sobolev spaces defined on $\Omega$ (see [Mantero-Zappa]). Complementary series representations are not weakly contained in the regular representation, but they too are realized on $\Omega$. (The two endpoints representations, $\pi_{i / 2}$ and $\pi_{\pi / \log q+i / 2}$ are characters, respectively the trivial character and the character with value -1 on each generator. In these cases the space of functions on $\Omega$ devolves into the space of constant functions.) Other known irreducible unitary representations of $\Gamma$ correspond to other measures on $\Omega$. For example, the measure $\nu$ given above depends on the choice of generators. If a new choice of generators gives a different spherical series, it also gives a different measure, mutually singular with $\nu$. The factor $\left|d \nu\left(g^{-1} \omega\right) / d \nu(\omega)\right|^{1 / 2+i s}$ above is called the cocycle. For many examples the cocycle, always a function of $g$ and $\omega$, cannot be expressed as a power of a Radon-Nikodym derivative.

The earliest studied irreducible representations were those of [Yoshizawa]. 
Obtained by inducing from a copy of $\mathbf{Z}$ inside $\Gamma$, they can be realized on $L^{2}\left(\Omega, \nu^{\prime}\right)$ where $\nu^{\prime}$ is an atomic measure.

It is possible to construct irreducible unitary representations of $\Gamma$ which are realized on spaces of vector-valued functions on $\Omega$. Let $\pi_{F}$ be a finite dimensional irreducible unitary representation of $\Gamma$, acting on $\mathscr{H}_{F}$, and let $\pi_{s}$ be in the spherical series. According to [Cowling-Steger] the tensor product $\pi_{s} \otimes \pi_{F}$, which can easily be realized on $L^{2}\left(\Omega, \mathscr{H}_{F}, \nu\right)$, is an irreducible representation.

Given the spherical principal series representation $\pi_{s}$ as an abstract representation, how might we reconstruct its realization on the boundary? First, choose a particular vector $v_{1}$ in the representation space $\mathscr{H}_{s}$, and identify $\mathscr{H}_{s}$ with a space of functions on $\Gamma$, identifying $v \in \mathscr{H}_{s}$ with the matrix coefficent $f_{v}$,

$$
f_{v}(\gamma)=\left\langle v, \pi_{s}(\gamma) v_{1}\right\rangle
$$

This identification intertwines $\pi_{s}$ with the ordinary action of $\Gamma$ by left translations. We will cheat and suppose that we have been so fortunate as to choose for $v_{1}$ the function identically 1 on $\Omega$. Second, identify the function $f_{v}$ with a certain function $F_{v}(\omega)$ defined on $\Omega$. Fix $\omega \in \Omega$ given by the half-geodesic $\left(x_{j}\right)_{j=0}^{\infty}$ starting at $e$. For fixed $v \in \mathscr{H}_{s}$ we find

$$
f_{v}\left(x_{j}\right)=c_{s}(\omega)\left(q^{-j}\right)^{\frac{1}{2}+i s}+c_{-s}(\omega)\left(q^{-j}\right)^{\frac{1}{2}-i s}+o\left(q^{-j}\right)^{\frac{1}{2}}
$$

or, more precisely, this holds for almost every $\omega \in \Omega$. (A slightly different asymptotic form holds if $s=0$ or $s=\pi / \log q$.) Let

$$
F_{v}(\omega)=c_{s}(\omega) \text { and } F_{v}^{\prime}(\omega)=c_{-s}(\omega)
$$

We may realize $\mathscr{H}_{s}$ as a space of functions on $\Omega$ by identifying $v \in \mathscr{H}_{s}$ with $F_{v}(\omega)$ (or alternatively with $F_{v}^{\prime}(\omega)$ ). The $F_{v}$-realization gives us back, up to a constant factor, the identification of $\mathscr{H}_{s}$ with $L^{2}(\Omega, \nu)$ which we used above to define $\pi_{s}$. The $F_{v}^{\prime}$-realization gives an essentially different identification of $\mathscr{H}_{s}$ with $L^{2}(\Omega, \nu)$.

Such a construction might conceivably apply to an arbitrary irreducible representation $(\pi, \mathscr{H})$ of $\Gamma$, provided that $\pi$ is weakly contained in the regular representation of $\Gamma$. However, there are many difficulties. Fixing $v_{1} \in \mathscr{H}$ one can identify $v \in \mathscr{H}$ with the matrix coefficient

$$
f_{v}(\gamma)=\left\langle v, \pi(\gamma) v_{1}\right\rangle .
$$

Even for the case just considered, $\pi=\pi_{s}$, the simple asymptotic form (1) would not have held if $v_{1}$ had not been chosen very carefully. For a general representation $\pi$, one does not know how to choose $v_{1}$, nor what sort of asymptotic form the functions $f_{v}$ are supposed to have. Nonetheless, one hopes to construct vector-valued functions $F_{v}$ on $\Omega$ which reflect the asymptotic behavior of the $f_{v}$. If $\omega=\left(e, x_{1}, x_{2}, \ldots\right)$ is a geodesic, then it is unreasonable to hope, in the general case, that the values $f_{v}\left(x_{j}\right)$ will determine $F(\omega)$. At the very least, values $f_{v}(y)$ for $y$ within fixed distance of the geodesic are required. 
Here are some conjectures which we hope will lead to a canonical way of realizing $\pi$ as acting on a space of vector-valued functions on $\Omega$. As above, $(\pi, \mathscr{H})$ should be irreducible, unitary, and weakly contained in the regular representation. For $x \in \Gamma$, let $|x|$ be the length of the reduced word expression for $x$. First, we conjecture

$$
\lim _{\varepsilon \rightarrow 0} \frac{\sum_{x \in \Gamma}\left\langle v_{1}, \pi(x) v_{2}\right\rangle \overline{\left\langle w_{1}, \pi(x) w_{2}\right\rangle} \exp (-\varepsilon|x|)}{\sum_{x \in \Gamma}\left|\left\langle u_{0}, \pi(x) u_{0}\right\rangle\right|^{2} \exp (-\varepsilon|x|)}=\left\langle v_{1}, w_{1}\right\rangle \overline{\left\langle v_{2}, w_{2}\right\rangle}
$$

assuming $\left\|u_{0}\right\|=1$. Second, we conjecture that the measures $\nu_{v, w}^{\varepsilon}$ on $\Gamma$ given by

$$
\nu_{v, w}^{\varepsilon}(y)=\frac{\left\langle v, \pi(y) u_{0}\right\rangle \overline{\left\langle w, \pi(y) u_{0}\right\rangle} \exp (-\varepsilon|x|)}{\sum_{x \in \Gamma}\left|\left\langle u_{0}, \pi(x) u_{0}\right\rangle\right|^{2} \exp (-\varepsilon|x|)}
$$

converge weakly to measures $\nu_{v, w}$ on $\Omega$, treating $\Gamma \cup \Omega$ as a single compact Hausdorff space. From the measures $\nu_{v, w}$ it should be possible to construct a direct integral of Hilbert spaces on $\Omega$, that is a Hilbert space bundle on $\Omega$, together with an action of $\Gamma$ on the bundle and an inclusion of $\mathscr{H}$ into the direct integral, that is, into the space of square integrable sections of the bundle. Without carrying this program through in full detail for any representation, we have calculated the apparent outcome in a number of cases. Strikingly, the full direct integral over $\Omega$ consists always of at most four irreducible components, frequently two irreducible components; in the case of the spherical principal series the two components are both equivalent to $\pi$, one giving the $F_{v}$-realization and the other the $F_{v}^{\prime}$-realization. Our third conjecture, less precise than the other two, is that this construction is robust with respect to the choice of $u_{0}$ and with respect to the choice of generators. Indeed, the generators enter into this construction in only one way, namely the determination of the length function on $\Gamma$. Any reasonable length function should yield the same realization on the boundary.

Return to the hypotheses of the theorem. We are given one matrix coefficient $\phi$ of some irreducible unitary representation $\pi$, and given that $\phi$ is invariant with respect to

$$
K_{N}=\{g \in \operatorname{Aut}(\mathscr{T}) ; g x=x \text { whenever } d(x, e) \leq N\} .
$$

This suggests that the measure on the boundary should be invariant with respect to $K_{N}$, which means it is in the same measure class as $\nu$, the $K_{0}$-invariant measure. Moreover, if $\left(x_{j}\right)_{j=0}^{\infty}$ is a geodesic from $e$ to some point of $\Omega$, then once the first $N$ steps are fixed, $\phi\left(x_{j}\right)$ depends only on $j$. Fixing those first $N$ steps, we can perform a Fourier or more properly a Laplace transform of $\phi\left(x_{j}\right)$ with respect to $j$, and expect the values of this transform to reflect the asymptotic behavior of $\phi\left(x_{j}\right)$. Indeed, comparison with (1) suggests that the individual coefficients of this Laplace transform correspond to subrepresentations of $\pi$ in the spherical series. Since $\pi$ is irreducible, $\pi$ should actually be equal to some member of the spherical series. The proof which follows reflects this approach, 
though it can be understood independently of the motivation. Observe that it is the spectral theorem (for bounded selfadjoint operators) which is called upon to effect the necessary Laplace transform in a controllable way.

\section{Proof of Theorem}

We are given an irreducible unitary representation, $\pi$, of $\Gamma$ acting on a Hilbert space, $\mathscr{H}$, and given two nonzero vectors $v_{0}, v_{1} \in \mathscr{H}$. The principal hypothesis is that

$$
\phi(x)=\left\langle v_{0}, \pi(x) v_{1}\right\rangle
$$

is $K_{N}$-invariant when considered as a function of the vertices of $\mathscr{T}$. As mentioned above, for $x \in \Gamma$, we let $|x|$ be the length of the reduced word for $x$ in terms of $\left\{a_{j}\right\}_{j=1}^{r}$. Alternatively, $|x|$ is the distance from $e$ to $x$ in the tree $\mathscr{T}$. Let

$$
\mu=\sum_{|y|=1} \delta_{y}
$$

be the characteristic function of the set of generators and inverses of generators. For any function $f: \Gamma \rightarrow \mathbf{C}$ define

$$
(A f)(x)=\sum_{d(x, z)=1} f(z)=\sum_{|y|=1} f(x y) .
$$

We have

$$
(A \phi)(x)=\sum_{|y|=1} \phi(x y)=\sum_{|y|=1}\left\langle v_{0}, \pi(x y) v_{1}\right\rangle=\left\langle v_{0}, \pi(x) \pi(\mu) v_{1}\right\rangle .
$$

It follows that if $Q$ is any polynomial,

$$
(Q(A) \phi)(x)=\left\langle v_{0}, \pi(x) Q(\pi(\mu)) v_{1}\right\rangle .
$$

Since $A$ preserves the space of $K_{N}$-invariant functions, the matrix coefficient between $v_{0}$ and $Q(\pi(\mu)) v_{1}$ is $K_{N}$-invariant. Now let $Q$ be any continuous function on $s p=\operatorname{spec}(\pi(\mu))$ and let $Q_{j}$ be a sequence of polynomials approaching $Q$. Then

$$
\left\langle v_{0}, \pi(x) Q(\pi(\mu)) v_{1}\right\rangle=\lim _{j \rightarrow \infty}\left\langle v_{0}, \pi(x) Q_{j}(\pi(\mu)) v_{1}\right\rangle
$$

is again $K_{N}$-invariant. Thus, we are at liberty to substitute $Q(\pi(\mu)) v_{1}$ for $v_{1}$, so long as the new vector is nonzero.

The spectral decomposition of $v_{1}$ with respect to the operator $\pi(\mu)$ will give a decomposition of $\phi$ into eigenfunctions of $A$. Let $\mathscr{H}^{(0)}$ be the closed linear span of $\left\{\pi(\mu)^{m} v_{1}\right\}_{m=0}^{\infty}$. The spectral theorem identifies $\mathscr{H}^{(0)}$ with $L^{2}(s p, d m(\lambda))$ where $s p$ denotes the spectrum of $\pi(\mu)$ and $d m$ is the spectral measure of $v_{1}$ with respect to $\pi(\mu)$. Under this identification $v_{1}$ corresponds to the function 1 and $\pi(\mu)^{n} v_{1}$ corresponds to the function $\lambda^{n}$. For each $x \in \Gamma$, define $\Phi_{x}: \mathscr{H}^{(0)} \rightarrow \mathbf{C}$ by

$$
\Phi_{x}(u)=\left\langle\pi\left(x^{-1}\right) v_{0}, u\right\rangle .
$$


Since $\Phi_{x}$ is a continuous antilinear functional on $\mathscr{H}^{(0)}$, there exists $\phi_{x} \in$ $L^{2}(s p, d m(\lambda))$ such that $\Phi_{x}$ is realized by

$$
\Phi_{x}(\psi)=\int_{s p} \phi_{x}(\lambda) \overline{\psi(\lambda)} d m(\lambda)
$$

Thus

$$
\phi(x)=\Phi_{x}\left(v_{1}\right)=\int_{s p} \phi_{x}(\lambda) d m(\lambda)
$$

and

$$
\left(A^{n} \phi\right)(x)=\Phi_{x}\left(\pi(\mu)^{n} v_{1}\right)=\int_{s p} \phi_{x}(\lambda) \lambda^{n} d m(\lambda) .
$$

Write $\phi_{\lambda}(x)$ for $\phi_{x}(\lambda)$. For any $n \geq 0$

$$
\begin{aligned}
\int_{s p}\left(A \phi_{\lambda}\right)(x) \lambda^{n} d m(\lambda) & =\sum_{|y|=1} \int_{s p} \phi_{\lambda}(x y) \lambda^{n} d m(\lambda)=\sum_{|y|=1}\left(A^{n} \phi\right)(x y) \\
& =\left(A^{n+1} \phi\right)(x)=\int_{s p} \phi_{\lambda}(x) \lambda^{n+1} d m(\lambda) .
\end{aligned}
$$

Since the linear span of the powers $\lambda^{n}$ is dense in $L^{2}(s p, d m(\lambda)), A \phi_{\lambda}(x)=$ $\lambda \phi_{\lambda}(x)$ for almost every $\lambda$. Those $\phi_{\lambda}$ which do not satisfy $A \phi_{\lambda}=\lambda \phi_{\lambda}$ can be replaced by zero. Thus

$$
\phi(x)=\int_{s p} \phi_{\lambda}(x) d m(\lambda) \text { where } A \phi_{\lambda}=\lambda \phi_{\lambda} .
$$

Next we show that (almost all) the $\phi_{\lambda}$ are $K_{N}$-invariant. Let $k \in K_{N}$. Identifying $x \in \Gamma$ as a vertex of $\mathscr{T}$, let $k(x) \in \Gamma$ be the image of $x$ under $k$. For $n \geq 0$

$$
\int_{s p} \phi_{\lambda}(k(x)) \lambda^{n} d m(\lambda)=\left(A^{n} \phi\right)(k(x))=\left(A^{n} \phi\right)(x)=\int_{s p} \phi_{\lambda}(x) \lambda^{n} d m(\lambda) .
$$

This holds because the $K_{N}$-invariance of $\phi$ implies that of $A^{n} \phi$. Again, since the span of the $\lambda^{n}$ is dense in $L^{2}(s p, d m(\lambda))$, it follows that $\phi_{\lambda}(k(x))=\phi_{\lambda}(x)$ for almost every $\lambda$. There are only countably many pairs $(x, k(x))$, so we may assume each of the $\phi_{\lambda}$ is $K_{N}$-invariant.

The spectrum, $s p$, of $\pi(\mu)$, is contained in the spectrum of $\mu$ as an element of $\ell^{1}(\Gamma)$. This spectrum is obviously inside $\left\{\lambda \in \mathbf{C} ;|\lambda| \leq\|\mu\|_{1}=2 r\right\}$. Since $s p$ is real, $s p \subseteq[-2 r, 2 r]$. Suppose that $-2 r \in s p$ and $m\{-2 r\}>0$. Then there exists $w$ in $\mathscr{H}^{(0)}$ such that $\pi(\mu) w=-2 r w$, i.e. $w$ such that $\sum_{|y|=1} \pi(y) w=-2 r w$. Since there are exactly $2 r$ terms in the sum it must be that $\pi\left(a_{j}\right) w=-w$ for each generator $a_{j}$. Thus $\mathbf{C} w$ is an invariant subspace of $\mathscr{H}$, hence all of $\mathscr{H}$. In fact, $\pi$ must be equivalent to the endpoint spherical series representation $\pi_{\pi / \log q+i / 2}$. Similarly, if $m\{2 r\}>0, \pi$ is equivalent to $\pi_{i / 2}$. If, on the other hand, $m\{ \pm 2 r\}$ is zero, then the support of $d m$ has some intersection with the open interval $(-2 r, 2 r)$. Replace $v_{1}$ with $Q(\pi(\mu)) v_{1}$ 
where $Q$ is a continuous function on $[-2 r, 2 r]$ with (compact) support completely contained in the open interval and large enough so that $Q(\pi(\mu)) v_{1} \neq 0$. Having done this, suppose for the rest of the proof that $\operatorname{supp}(\mu) \subseteq(-2 r, 2 r)$.

Next we will show that for each $\lambda, \phi_{\lambda}(x)$ is a matrix coefficient of $\pi_{s}$ where

$$
\lambda=q^{\frac{1}{2}+i s}+q^{\frac{1}{2}-i s} \text {. }
$$

Real values of $s$ correspond to $\lambda$ in the range $[-2 \sqrt{q}, 2 \sqrt{q}]$, values of $s$ with $\operatorname{Re}(s)=0$ correspond to $\lambda$ in the range $[2 \sqrt{q}, 2 r]$, and values of $s$ with $\operatorname{Re}(s)=\pi / \log q$ correspond to $\lambda$ in the range $[-2 r,-2 \sqrt{q}]$. Let $\mathscr{H}_{s}$ be the representation space of $\pi_{s}$, realized as a space of functions on $\Omega$. Let $1_{s} \in \mathscr{H}_{s}$ be the $K_{0}$-invariant vector given by the function identically one on $\Omega$. Normalize the inner product on $\mathscr{H}_{s}$ so that $\mathbf{1}_{s}$ has length 1 . For $u \in \mathscr{H}_{s}$ let the Poisson transform of $u$ (see [Mantero-Zappa]) be given by

$$
\left(\mathscr{P}_{s} u\right)(g)=\left\langle u, \pi_{s}(g) \mathbf{1}_{s}\right\rangle \text { for } g \in \operatorname{Aut}(\mathscr{T}) .
$$

The Poisson transform intertwines $\pi_{s}$ with the left-regular action of $\operatorname{Aut}(\mathscr{T})$. Since $1_{s}$ is $K_{0}$-invariant, $\mathscr{P}_{s} u$ is defined on the coset space $\operatorname{Aut}(\mathscr{T}) / K_{0}$, which is to say that $\mathscr{P}_{s} u$ can be thought of as a function on the vertices of $\mathscr{T}$. In particular, the restriction of $\mathscr{P}_{s} u$ to $\Gamma$ determines $\mathscr{P}_{s} u$. Since $\mathbf{1}_{s}$ is a $\lambda$ eigenvector of $\pi_{s}(\mu), \mathscr{P}_{s} u$ is a $\lambda$-eigenfunction of $A$. The Helgason theorem of [Mantero-Zappa], described below, implies that every $K_{N}$-invariant $\lambda$ eigenfunction of $A$ is in the range of $\mathscr{P}_{s}$. Choose $u_{\lambda} \in \mathscr{H}_{s}$ so that $\mathscr{P}_{s} u_{\lambda}=\phi_{\lambda}$. Then

$$
\phi_{\lambda}(x)=\left\langle u_{\lambda}, \pi(x) \mathbf{1}_{s}\right\rangle .
$$

For any vertex $x$ of $\mathscr{T}$, let $[e, x]$ be the geodesic from $e$ to $x$. Similarly, for $\omega \in \Omega$, let $[e, \omega]$ be the infinite geodesic from $e$ to $\omega$. Define $D(x, \omega)$ as the distance from $e$ of the last vertex common to $[e, x]$ and $[e, \omega]$. Define the Poisson kernel, $P(x, \omega)$, by $P(x, \omega)=q^{-d(x, e)+2 D(x, \omega)}$. Then the Poisson transform is given concretely by

$$
\left(\mathscr{P}_{s} u\right)(x)=\int_{\Omega} P(x, \omega)^{\frac{1}{2}-i s} u(\omega) d \nu(\omega) .
$$

For fixed $\omega, P(x, \omega)^{\frac{1}{2}-i s}$ is a $\lambda$-eigenfunction of $A$. For a fixed $x, P(x, \omega)$ belongs to $\mathscr{K}(\Omega)$, the space of locally constant functions on $\Omega$. For $U \in$ $\mathscr{K}^{\prime}(\Omega)$ the algebraic dual of $\mathscr{K}(\Omega)$, set

$$
\left(\mathscr{P}_{s}^{\prime} U\right)(x)=U\left(P(x, \omega)^{\frac{1}{2}-i s}\right) .
$$

The Helgason theorem of [Mantero-Zappa] (see also [Kato 1 and 2]) asserts that $\mathscr{P}_{s}^{\prime}$ is an isomorphism from $\mathscr{K}^{\prime}(\Omega)$ to the space of all $\lambda$-eigenfunctions of $A$. Moreover, since $P(k(x), k(\omega))=P(x, \omega)$ for every $k \in K_{0}, \mathscr{P}_{s}^{\prime}$ intertwines the action of $K_{0}$ on $\mathscr{K}^{\prime}(\Omega)$ and the left-regular action of $K_{0}$ on the space of eigenfunctions. In particular, $K_{N}$-invariant eigenfunctions correspond to $K_{N^{-}}$ invariant functionals in $\mathscr{K}^{\prime}(\Omega)$. Furthermore, any $K_{N}$-invariant functional $U$ 
must be given by integration against $u(\omega) d \nu(\omega)$ where $u$ is some $K_{N}$-invariant function. All the $K_{N}$-invariant functions belong to $\mathscr{H}_{s}$, so every $K_{N}$-invariant $\lambda$-eigenfunction of $A$ is given as $\mathscr{P}_{s} u$ for some $u \in \mathscr{H}_{s}$.

Given a $K_{N}$-invariant function $u$ and given $\omega \in \Omega$, let $[e, \omega]=\left(x_{j}\right)_{j=0}^{\infty}$. Direct calculation gives

$$
\left(\mathscr{P}_{s} u\right)\left(x_{j}\right)=C_{s} u(\omega)\left(q^{-j}\right)^{\frac{1}{2}+i s}+C_{-s} u^{\prime}(\omega)\left(q^{-j}\right)^{\frac{1}{2}-i s} \text { for } j \geq N .
$$

Here we must exclude the special cases $s=0$ and $s=\pi / \log q$. The constant is

$$
C_{s}=\frac{\sqrt{q}}{q+1} \frac{q^{\frac{1}{2}-i s}-q^{-\frac{1}{2}+i s}}{q^{-i s}-q^{i s}},
$$

while $u^{\prime}$ is given by a complicated formula involving the values of $u$ at all points of $\Omega$. This formula is not part of the proof. Rather, it shows that $u_{\lambda}$ may be obtained by examining the asymptotic behavior of $\phi_{\lambda}(x)$ as $x$ approaches the boundary of the tree.

Here is an explicit formula which inverts $\mathscr{P}_{s}$ on the (finite-dimensional) space of $K_{N}$-invariant $\lambda$-eigenfunctions of $A$. Let $\omega \in \Omega$ and let $[e, \omega]=$ $\left(x_{j}\right)_{j=0}^{\infty}$. Then

$$
u(\omega)=\frac{q+1}{q}\left(q^{\frac{1}{2}+i s}\right)^{N} \frac{\left(\mathscr{P}_{s} u\right)\left(x_{N}\right) q^{\frac{1}{2}-i s}-\left(\mathscr{P}_{s} u\right)\left(x_{N-1}\right)}{q^{\frac{1}{2}-i s}-q^{-\frac{1}{2}+i s}} .
$$

This follows because $P\left(x_{N-1}, \omega^{\prime}\right)=q P\left(x_{N}, \omega^{\prime}\right)$ when $[e, \omega]$ and $\left[e, \omega^{\prime}\right]$ do not have the same first $N$ steps, while $P\left(x_{N}, \omega^{\prime}\right)=q^{N}$ and $P\left(x_{N-1}, \omega^{\prime}\right)=$ $q^{N-1}$ when $[e, \omega]$ and $\left[e, \omega^{\prime}\right]$ do have the same first $N$ steps. For $K_{N^{-}}$ invariant $u$, the norm of $u$ in $\mathscr{H}_{s}$ is equivalent to the norm of $u$ in $L^{2}(\Omega, d \nu)$ (see [Mantero-Zappa]). Of course, in the principal series, $\operatorname{Im} s=0$, these two norms are equal. The constant controlling this equivalence of norms depends on $N$ and $s$ and blows up as $s$ approaches $i / 2$ or $i / 2+\pi / \log q$, that is, as $\lambda$ approaches $\pm 2 r$. So long as we are working with fixed $N$ and with $\lambda$ in a compact subset of $(-2 r, 2 r)$, formula (2) gives

$$
\|u\| \leq C \sum_{|x| \leq N}\left|\left(\mathscr{P}_{s} u\right)(x)\right|
$$

for some constant $C$. In particular

$$
\left\|u_{\lambda}\right\| \leq C \sum_{|x| \leq N}\left|\phi_{\lambda}(x)\right|
$$

Thus

$$
\int_{s p}\left\|u_{\lambda}\right\|^{2} d m(\lambda) \leq C \int_{s p}\left(\sum_{|x| \leq N}\left|\phi_{\lambda}(x)\right|\right)^{2} d m(\lambda)<\infty .
$$

Let

$$
\mathscr{H}^{\prime}=\int_{s p}^{\oplus} \mathscr{H}_{s(\lambda)} d m(\lambda), \quad v_{0}^{\prime}=\int_{s p}^{\oplus} u_{\lambda} d m(\lambda), \quad v_{1}^{\prime}=\int_{s p}^{\oplus} \mathbf{1}_{s(\lambda)} d m(\lambda) .
$$


Let $\pi^{\prime}$ denote the obvious direct integral representation of $\Gamma$ on $\mathscr{H}^{\prime}$. Then

$$
\begin{aligned}
\left\langle v_{0}^{\prime}, \pi^{\prime}(x) v_{1}^{\prime}\right\rangle & =\int_{s p}\left\langle u_{\lambda}, \pi_{s(\lambda)}(x) \mathbf{1}_{s(\lambda)}\right\rangle d m(\lambda) \\
& =\int_{s p} \phi_{\lambda}(x) d m(\lambda)=\phi(x)=\left\langle v_{0}, \pi(x) v_{1}\right\rangle .
\end{aligned}
$$

The result now follows from two standard lemmas (applicable to all locally compact groups).

Lemma 1. Suppose $\pi\left(\pi^{\prime}\right)$ is an irreducible unitary (a unitary) representation of $\Gamma$ on $\mathscr{H}\left(\mathscr{H}^{\prime}\right)$. Suppose there exist nonzero vectors $v_{0}, v_{1} \in \mathscr{H}$ and $v_{0}^{\prime}$, $v_{1}^{\prime} \in \mathscr{H}^{\prime}$ such that $\left\langle v_{0}, \pi(x) v_{1}\right\rangle=\left\langle v_{0}^{\prime}, \pi^{\prime}(x) v_{1}^{\prime}\right\rangle$ for $x \in \Gamma$. Then $\pi$ is a subrepresentation of $\pi^{\prime}$.

Lemma 2. An irreducible subrepresentation of a direct integral of irreducible representations is equivalent to one of the integrand representations.

To prove Lemma 1 we must construct a nonzero map from $\mathscr{H}$ to $\mathscr{H}^{\prime}$ which intertwines $\pi$ and $\pi^{\prime}$. Define

$$
\mathscr{H}^{\prime \prime}=\text { closed-span }\left(\pi(x) v_{1}, \pi^{\prime}(x) v_{1}^{\prime}\right)_{x \in \Gamma}
$$

inside $\mathscr{H} \oplus \mathscr{H}^{\prime}$. Let $P\left(P^{\prime}\right)$ denote the obvious projection from $\mathscr{H}^{\prime \prime}$ to $\mathscr{H}\left(\mathscr{H}^{\prime}\right)$. If $P^{\prime} P^{*}$ is nonzero then it is the required intertwining map. Since $P P^{*}$ is an intertwiner for the irreducible representation $\pi$, it is a scalar, obviously nonzero. Hence if $P^{\prime} P^{*}(w)=0$ for some nonzero $w \in \mathscr{H}$, then $\mathscr{H}^{\prime \prime}$ contains the element $(w, 0)$.

To say that $(w, 0)$ is in $\mathscr{H}^{\prime \prime}$ is to say that for any $\varepsilon>0$ we can find constants $\left(c_{x}\right)_{x \in \Gamma}$ such that

For any $y \in \Gamma$

$$
\left\|w-\sum c_{x} \pi(x) v_{1}\right\|<\varepsilon \text { and }\left\|\sum c_{x} \pi^{\prime}(x) v_{1}^{\prime}\right\|<\varepsilon .
$$

$$
\begin{aligned}
\left|\left\langle\pi(y) v_{0}, w\right\rangle\right| & \leq \varepsilon\left\|v_{0}\right\|+\left|\left\langle\pi(y) v_{0}, \sum c_{x} \pi(x) v_{1}\right\rangle\right| \\
& =\varepsilon\left\|v_{0}\right\|+\left|\left\langle\pi^{\prime}(y) v_{0}^{\prime}, \sum c_{x} \pi^{\prime}(x) v_{1}^{\prime}\right\rangle\right| \\
& \leq \varepsilon\left\|v_{0}\right\|+\varepsilon\left\|v_{0}^{\prime}\right\|
\end{aligned}
$$

Thus for each $y,\left\langle\pi(y) v_{0}, w\right\rangle=0$. Since $\pi$ is irreducible, $w$ must be 0 , a contradiction.

To prove Lemma 2, take some vector in the representation space of the irreducible subrepresentation, and write the corresponding positive definite function as an integral of positive definite functions coming from the integrand representations. Then almost all of the integrand positive definite functions are multiples of the irreducible positive definite function which is their integral. There must be at least one of the integrand positive definite functions which is a positive multiple of the integral, and the corresponding integrand representation is equivalent to the subrepresentation with which we started. 


\section{REFERENCES}

[Angelini] F. Angelini, Rappresentazioni di un gruppo libero associate ad una passegiata a caso, undergraduate thesis submitted at the Università degli Studi di Roma "La Sapienza," 1989.

[Bishop-Steger] C. Bishop and T. Steger, Representation theoretic rigidity in $\operatorname{PSL}(2, \mathbb{R})$, preprint.

[Cartier 1] P. Cartier, Géométrie et analyse sur les abres, Sem. Bourbaki vol. 1971/1972, Exposès 400-417, Lecture Notes in Math. vol. 317, Springer-Verlag, Berlin, 197, pp. 123-140.

[Cartier 2] __, Fonctiones harmoniques sur les arbres, Sympos. Math. 9 (1972), 203-270.

[Cartier 3] __ Harmonic analysis on trees, Proc. Sympos. Pure Math., vol. 9, Amer. Math. Soc., Providence, RI, 1972, pp. 419-424.

[Cecchini-Figà-Talamanca] C. Cecchini and A. Figà-Talamanca, Projections of uniqueness for $L^{p}(G)$, Pacific J. Math. 51 (1974), 37-47.

[Culler-Morgan] M. Culler and J. Morgan, Groups acting on $\mathbb{R}$-trees, Proc. London Math. Soc. 55 (1985), 571-604.

[Cowling-Steger] M. Cowling and T. Steger, Irreducibility of restrictions of unitary representations to lattices, preprint.

[Dunford-Schwartz] N. Dunford and J. T. Schwartz, Linear operators, Part II, Interscience, New York, 1963.

[Figà-Talamanca-Picardello 1] A. Figà-Talamanca, and A. M. Picardello, Spherical functions and harmonic analysis on free groups, J. Funct. Anal. 47 (1982), 281-304.

[Figà-Talamanca-Picardello 2] __, Restriction of spherical representations of $P G L\left(2, Q_{p}\right)$ to a discrete subgroup, Proc. Amer. Math. Soc. 91 (1984), 405-408.

[Figà-Talamanca-Picardello 3] ___, Harmonic analysis on free groups, Lecture Notes in Pure and Appl. Math., vol. 87, Marcel Dekker, New York, 1983.

[Figà-Talamanca-Steger 1] A. Figà-Talamanca and T. Steger, Harmonic analysis on trees, Sympos. Math. 29 (1987), 163-182.

[Figà-Talamanca-Steger 2] __ Harmonic analysis for anisotropic random walks on homogeneous trees, Mem. Amer. Math. Soc. (to appear).

[Haagerup] U. Haagerup, An Example of a nonnuclear $C^{\star}$-algebra which has the metric approximation property, Invent. Math 50 (1979), 279-293.

[Helgason] S. Helgason, A duality for symmetric spaces with applications to group representations, Adv. in Math. 5 (1970), 1-154.

[Kajiwara] T. Kajiwara, On irreducible decompositions of the regular representation of free groups, Boll. Un. Mat. Ital. 6 (1985), 425-431.

[Kato 1] Shin-ichi Kato, Irreducibility of principal series representations for Hecke algebras of affine type, J. Fac. Sci. Univ. Tokyo Sect. IA Math. 28 (1981), 929-943.

[Kato 2] __, On eigenspaces of the Hecke algebra with respect to a good maximal compact subgroup of a p-adic reductive group, Math. Ann. 257 (1981), 1-7.

[Mantero-Zappa] A. M. Mantero and A. Zappa, The Poisson transform on free groups and uniformly bounded representations, J. Funct. Anal. 51 (1983), 372-399.

[OI'shanskii 1] G. I. Ol'shanskii, Representations of groups of automorphisms of trees, Uspekhi Mat. Nauk 30 (1975), 169-170. (Russian)

[Ol'shanskii 2] __ Classification of irreducible representations of groups of automorphisms of Bruhat-Tit trees, Funct. Anal. Appl. 11 (1977), 26-34.

[Pytlik] T. Pytlik, Radial functions on free groups and a decomposition of the regular representation into irreducible components, J. Reine Angew. Math. 326 (1981), 124-135.

[Pytlik-Swarc] T. Pytlik and R. Swarc, An analytic family of uniformly bounded representations of free groups, Acta Math. 157 (1986), 287-309.

[Rudin] W. Rudin, Functional analysis, McGraw-Hill, New York, 1973. 
[Steger] T. Steger, Finite reducibility of random walk representations of free groups, in preparation. [Yoshizawa] H. Yoshizawa, Some remarks on unitary representations of the free group, Osaka Math. J. 3 (1951), 55-63.

Dipartimento Matematica, Università di Milano, via C. Saldini 50, 20133 Milano, ITALIA

Department of Mathematics, University of Chicago, 5734 S. University Avenue, Chicago, Illinois 60637 